Author manuscript

J Chromatogr B Analyt Technol Biomed Life Sci. Author manuscript; available in PMC 2017 December 01.

Published in final edited form as: J Chromatogr B Analyt Technol Biomed Life Sci. 2016 December 01; 1038: 57-62. doi:10.1016/ j.jchromb.2016.10.026.

\title{
Development of HPLC and LC-MS/MS methods for the analysis of ivacaftor, its major metabolites and lumacaftor in plasma and sputum of cystic fibrosis patients treated with ORKAMBI or KALYDECO
}

\author{
Elena K. Schneider ${ }^{1}$, Felisa Reyes-Ortega ${ }^{1}$, John W. Wilson ${ }^{2,3}$, Tom Kotsimbos ${ }^{2,3}$, Dominic \\ Keating ${ }^{2,3}$, Jian $\mathbf{L i}^{1,4}$, and Tony Velkov ${ }^{1, \dagger}$ \\ ${ }^{1}$ Drug Delivery, Disposition and Dynamics, Monash Institute of Pharmaceutical Sciences; Monash \\ University, Parkville, Victoria 3052, Australia \\ ${ }^{2}$ Department of Medicine, Monash University, Alfred Hospital, Commercial Road, Melbourne, \\ Victoria 3004, Australia \\ ${ }^{3}$ Department of Allergy, Immunology and Respiratory Medicine, Alfred Hospital, Commercial \\ Road, Melbourne, Victoria 3004, Australia \\ ${ }^{4}$ Monash Biomedicine Discovery Institute, Department of Microbiology, Monash University, \\ Clayton, Victoria 3800, Australia
}

\section{Abstract}

ORKAMBI (ivacaftor-lumacaftor [LUMA]) and KALYDECO (ivacaftor; IVA) are two new breakthrough cystic fibrosis (CF) drugs that directly modulate the activity and trafficking of the defective CFTR underlying the CF disease state. Currently, no therapeutic drug monitoring assays exist for these very expensive, albeit, important drugs. In this study, for the first time HPLC and LC-MS methods were developed and validated for rapid detection and quantification of IVA and its major metabolites hydroxymethyl-IVA M1 (active) and IVA-carboxylate M6 (inactive); and LUMA in the plasma and sputum of CF patients. With a mobile phase consisting of acetonitrile/ water: $0.1 \%$ formic acid $(60: 40 \mathrm{v} / \mathrm{v})$ at a flow rate of $1 \mathrm{~mL} / \mathrm{min}$, a linear correlation was observed over a concentration range from 0.01 to $10 \mu \mathrm{g} / \mathrm{mL}$ in human plasma (IVA R ${ }^{2}>0.999$, IVA M1 R ${ }^{2}>$ 0.9961 , IVA M6 $\mathrm{R}^{2}>0.9898$, LUMA $\mathrm{R}^{2}>0.9954$ ). The assay was successfully utilized to quantify the concentration of LUMA, IVA, M1 and M6 in the plasma and sputum of CF patients undergoing therapy with KALYDECO (IVA $150 \mathrm{mg} / \mathrm{q} 12 \mathrm{~h}$ ) or ORKAMBI (200 mg/q12 h LUMA-125 mg/q12 h IVA). The KALYDECO patient exhibited an IVA plasma concentration of $0.97 \mu \mathrm{g} / \mathrm{mL}$ at $2.5 \mathrm{~h}$ post dosage. M1 and M6 plasma concentrations were $0.50 \mu \mathrm{g} / \mathrm{mL}$ and 0.16 $\mu \mathrm{g} / \mathrm{mL}$, respectively. Surprisingly, the ORKAMBI patient displayed very low plasma concentrations of IVA $(0.06 \mu \mathrm{g} / \mathrm{mL})$ and M1 $(0.07 \mu \mathrm{g} / \mathrm{mL})$. The M6 concentrations $(0.15 \mu \mathrm{g} / \mathrm{mL})$ were comparable to those of the KALYDECO patient. However, we observed a relatively high plasma concentration of LUMA $(4.42 \mu \mathrm{g} / \mathrm{mL})$. This reliable and novel method offers a simple and sensitive approach for therapeutic drug monitoring of KALYDECO and ORKAMBI in plasma and sputum. The introduction of the assay into the clinical setting will facilitate pharmacokinetics/ 
pharmacodynamic analysis and assist clinicians to develop more cost effective and efficacious dosage regimens for these breakthrough CF drugs.

\section{Keywords}

Ivacaftor; lumacaftor; LC/MS; cystic fibrosis; plasma; sputum

\section{Introduction}

Cystic Fibrosis is an autosomal recessive life limiting disease that is caused by defective or deficient cystic fibrosis transmembrane conductance regulator (CFTR) protein [1]. The disease affects the exocrine mucus glands in the lung, liver, pancreas, and intestines causing progressive multi-system failure such as loss of lung function and pancreatic insufficiency [2-6]. KALYDECO (ivacaftor, IVA) is the first FDA-approved CFTR modulator, with evidenced clinical efficacy producing a significant improvement in the lung function of $\mathrm{CF}$ patients with the G551D-CFTR mutation (found in 4-5\% of CF cases) [7]. More specifically, IVA functions as a CFTR potentiator producing an increased CFTR channel open probability to enhance chloride influx [8-10]. ORKAMBI is a novel combination treatment that combines the potentiator function of IVA with lumacaftor (LUMA), a CFTR corrector which improves the surface trafficking of CFTR in CF patients bearing a homozygous F508del mutation, expanding the therapeutic window to the broader CF patient collective ( 28\%) [11]. The annual cost per patient for both drugs is high $(\$ 259,000$ $\$ 311,000$ USD), and presently their manufacturer Vertex has a market monopoly as these are the only drugs on the market available for their indication [12]. The clinical efficacy of ORKAMBI versus the cost of therapy has been brought into question due to potential antagonistic drug-drug interactions between IVA and LUMA that potentially limit the clinical efficacy of ORKAMBI [13, 14]. Additional factors that may limit ORKAMBI's clinical efficacy come from its less than ideal pharmacokinetic properties [15, 16]. IVA is extensively metabolized by cytochrome $\mathrm{P} 450$ enzymes, primarily to an active metabolite hydroxymethyl-IVA (M1) and an inactive form IVA-carboxylate (M6) [15, 17, 18]. LUMA on the other hand is not extensively metabolized and is largely excreted unchanged in the faeces [16]. Both IVA and LUMA are very hydrophobic molecules and are $99 \%$ bound to plasma proteins, which significantly limits the free (active) drug concentration [2, 14]. Presently, very little information is available concerning the steady-state plasma concentrations of IVA, M1, M6 and LUMA achievable in patients under the current recommended dosage regimen no methods for therapeutic drug monitoring have been reported so far. In this study, we have developed a reliable and simple LC-MS method for the quantification of IVA, its major metabolites M1, M6 and LUMA in plasma and sputum for therapeutic drug monitoring of CF patients receiving KALYDECO or ORKAMBI therapy. The implementation of the assay in clinical centers should assist clinicians to develop exposure-response relationships to maximize drug efficacy as well as to contain healthcare costs. 


\section{Material and Methods}

\subsection{Materials}

IVA and LUMA were purchased from SelleckChem, USA. IVA-carboxylate (Catalogue number 510242247CS) and hydroxymethyl-IVA (Catalogue number 510240849CS) were from Clearsynth (Canada) and were used as internal standards (IS) in methanol at $10 \mu \mathrm{g} / \mathrm{mL}$. Methanol (MeOH, LC-MS grade), acetonitrile (ACN, LC-MS grade) and formic acid were purchased from Sigma-Aldrich (Australia). Experiments were performed on a triplequadrupole Shimadzu 8030 LC-MS and a Nexera X2 Shimadzu HPLC (SPD-M30A detector) systems using a Waters C 8 column $(5 \mu \mathrm{m}, 3.9 \times 50 \mathrm{~mm}$ i.d.). Plasma was obtained from the Australian Red Cross. Plasma and sputum samples were collected from two compliant volunteers receiving ORKAMBI or KALYDECO therapy.

\subsection{Preparation of stock solutions and sample standards}

Two independent stock solutions of each analyte (IVA, M1, M6 and LUMA) were freshly prepared in methanol for each analytical run at $100 \mu \mathrm{g} / \mathrm{mL}$. Stock solutions of the internal standards (IS) were prepared in methanol at $10 \mu \mathrm{g} / \mathrm{mL}$. The IS working solutions were prepared by a further dilution of each IS stock solution with methanol. Eight calibration standards stock solutions of each analyte at $1,2.5,5,10,25,40,65$ and $80 \mu \mathrm{g} / \mathrm{mL}$ were prepared in methanol by serial dilution of the $100 \mu \mathrm{g} / \mathrm{mL}$ stock solutions. The LC-MS calibration standards were freshly prepared at the beginning of each analytical run by dilution of the calibration standards stock solutions into human plasma to achieve following concentrations: $0.01,0.025,0.05,0.1,0.2,0.5,1,2.5,5$ and $10 \mu \mathrm{g} / \mathrm{mL}$.

\subsection{Sample pre-treatment}

Plasma or sputum samples were vortex-mixed before sampling and an aliquot of $200 \mu \mathrm{L}$ was transferred into a $1.5 \mathrm{~mL}$ polypropylene microcentrifuge tube (VWR). Protein precipitation was achieved by an addition of $200 \mu \mathrm{L}$ of a mixture of $\mathrm{ACN} / 0.1 \%$ formic acid. The mixture was vortexed vigorously, then centrifuged at $10,000 \times g$ for $10 \mathrm{~min}$ (Eppendorf Centrifuge 5430) at room temperature. A $200 \mu \mathrm{L}$ aliquot of the supernatant was filtered through a 13$\mathrm{mm}$ syringe filter ( $0.45 \mu \mathrm{m}$ nylon, GRACE, USA) into an HPLC $1.5 \mathrm{~mL}$ vial [Phenomenex VEREX, 9mm, PP, 300 $\mu \mathrm{L}$, PTFE/Silicone septa]. An aliquot of $5 \mu \mathrm{L}$ was injected onto the HPLC column. The method validation procedure for the IVA assay was based on the FDA guidelines for bioanalytical method validation [19]. Partially method validation was performed including parameters listed in Table 1 as the aim was to develop a method that is easily accessible for hospital laboratories measuring all 3 or 4 compounds in one single assay.

\subsection{HPLC analysis}

Initial HPLC method development for the analysis of IVA and LUMA in biological matrices was performed on a Shimadzu Nexera X2 HPLC system. Analytes were separated on Waters $\mathrm{C} 8$ column $(3.9 \times 150 \mathrm{~mm}$ id, $5 \mu \mathrm{m})$ under isocratic conditions at $1 \mathrm{~mL} / \mathrm{min}$. The mobile phase consisted of $\mathrm{ACN} / \mathrm{H}_{2} \mathrm{O}: 0.1 \%$ formic acid mixture (60:40 v/v). Column temperature was set to $30^{\circ} \mathrm{C}$ and sample compartment temperature was set to $4^{\circ} \mathrm{C}$. The injection volume 
was $5 \mu \mathrm{L}$. Total run time was 13 min including the wash and equilibration steps.

Quantification of IVA (also M1 and M6) and LUMA was performed at $309 \mathrm{~nm}$ and $295 \mathrm{~nm}$, respectively.

\subsection{LC-MS/MS analysis}

The LC-MS/MS analysis of patient samples was performed on a Shimadzu 8030 LCMS system coupled with the 8030 triple quadrupole mass spectrometer. The mass spectrometer operated in a positive electrospray ionization mode. LCMS settings were: ion spray voltage $4.5 \mathrm{kV}$, collision energy $295.9 \mathrm{~V}$, nebulizing gas: nitrogen at $3 \mathrm{~L} / \mathrm{min}$; collision gas: argon; drying gas flow $20 \mathrm{~L} / \mathrm{min}$, lens voltage $\mathrm{Q} 3:-22 \mathrm{~V}$, desolvation temperature $250^{\circ} \mathrm{C}$ with a heat block temperature of $400^{\circ} \mathrm{C}$. The mobile phase flow was split before entering the mass spectrometer in ratio 2:1 (waste: MS inlet). Analytes were detected using multiple reaction monitoring (MRM). The ion transitions of $\mathrm{m} / \mathrm{z} 392.49 \rightarrow 393, \mathrm{~m} / \mathrm{z} 408.49 \rightarrow 409, \mathrm{~m} / \mathrm{z}$ $422.47 \rightarrow 423$ and $m / z \quad 452.40 \rightarrow 453$ were monitored for IVA, M1, M6 and LUMA, respectively.

\subsection{Calibration curves}

The LC-MS calibration curves were constructed before each analytical run using the relationship between the peak area ratios of IVA to IS and the calibration standard nominal concentrations of IVA (M1, M6 or LUMA). Linear least-squares regression analysis with weighting $1 / \mathrm{C}^{\wedge} 2$ was performed according to the reciprocal of concentrations (Shimadzu).

\subsection{Accuracy and precision}

The intra-day accuracy of each analyte was assessed with six independently prepared quality control (QC) samples on the same day at concentrations of $0.05,0.5$ and $8 \mu \mathrm{g} / \mathrm{mL}$. The interday accuracy was assessed with six independently prepared QC samples on three consecutive days. Accuracy and precision were calculated via relative standard deviation (RSD). For each QCs, the RSD values should be less than 15\% [20]. The precision of an analytical procedure expresses the closeness of agreement between a series of measurements obtained from multiple sampling of the same homogeneous sample under the prescribed conditions. Precision may be considered at three levels: repeatability, intermediate precision and reproducibility. In this study, the precision of our analytical procedure was expressed as relative standard deviation or coefficient of variation of a series of measurements. The relative standard deviation was calculated by taking the standard deviation of the sample set multiplied by $100 \%$ and dividing it by the sample set average. The relative standard deviation is expressed as percent:

$$
\% \mathrm{RSD}=\mathrm{s} / \mathrm{x} * 100 \%
$$

Being $\mathrm{s}$ the standard deviation and $\mathrm{x}$ the average of the three independent measurements.

To confirm the precision, the predicted \%RSD has to be compare with the experimental $\% \mathrm{RSD}$, as follows: 


\section{$\%$ RSD theoretical $=2 \exp (1-0.5 \log C)$}

Being $\mathrm{C}$ the real concentration of the sample.

Good inter-day precision: \%RSD exp $\leq \%$ RSD theoretical

Good intra-day precision: \%RSD exp $\leq \% \mathrm{RSD}$ theoretical/2

\subsection{Recovery}

The relative recovery of IVA, M1, M6 and LUMA was calculated by comparing the peak areas of the samples prepared in human plasma with those obtained from a set of quality control samples prepared in methanol. While the full information on the analytes' recovery from the matrix, that is the relative recovery and the extraction recovery, is beneficial and gives a better understanding of the closeness of the values determined to the true values; in our study the aim was to develop a 'fit for purpose method' with the view on further development and testing as required when larger sets of materials and matrices are available. To assess the applicability of the method for routine analysis, we utilised the commonly employed strategy for estimating relative recovery to obtain a reference point for the compounds behaviour in human plasma. Our data indicated a good relative recovery. The recovery was consistent for two different concentration levels indicating that matrix effect is not concentration dependant and would not affect the quantitation of samples at various concentrations of analytes. In future experiments with a larger cohort of patient samples (various stages of illness, co-medication etc) the effect of extraction recovery will be investigated as it might differ between samples. To explore further extraction currently exceeds the scope of this study but should ideally be explored in future.

\subsection{Patient samples}

Approval for ethics was obtained from Monash University Human Research Ethics Committee (MUHREC). Plasma and sputum samples were collected from two compliant patients: patient one treated with IVA $150 \mathrm{mg} / \mathrm{q} 12 \mathrm{~h}$ (KALYDECO) and patient two treated with $200 \mathrm{mg} / \mathrm{q} 12 \mathrm{~h}$ LUMA-125 mg/q12h IVA (ORKAMBI). Plasma samples were collected at $2.5 \mathrm{~h}$ after dosing at steady state (after $>2$ weeks of treatment). Sputum (approximately 2 $3 \mathrm{~mL}$ ) was collected at $2.5 \mathrm{~h}$ post-dosing. Plasma and sputum samples were treated as described above for LC/MS assays.

\section{Results and Discussion}

Our HPLC method provides excellent and reproducible separation of IVA, M1, M6 and LUMA within a short run time $(7.5 \mathrm{~min})$. Typical chromatograms are shown in Figures $1 \&$ 2. Optimized chromatographic resolution was obtained using a mobile phase of ACN/ $0.1 \%$ formic acid: $\mathrm{H}_{2} \mathrm{O}(60: 40, \mathrm{v} / \mathrm{v})$ with an isocratic elution on a Waters $\mathrm{C} 8$ column $(5 \mu \mathrm{m}$, $3.9 \times 150 \mathrm{~mm}$ ). A linear calibration range from 0.01 to $10 \mu \mathrm{g} / \mathrm{mL}$ was established for the LCMS assay and a range from $1 \mu \mathrm{g} / \mathrm{mL}$ to $80 \mu \mathrm{g} / \mathrm{mL}$ for HPLC (Figure 3). The LC retention times were as follows IVA $6.2 \mathrm{~min}$, M1 $2.4 \mathrm{~min}$, M6 $2.1 \mathrm{~min}$ and LUMA $5.4 \mathrm{~min}$ (Figure 1). In all blank plasma samples no interference with the retention time of either of the analytes 
was observed, nor was interference detected from the combination of IVA with LUMA. The method used to determine the endogenous interferences was the percentage of the area of the LOD for each analyte. Linearity of all of the calibration curves was observed (Figure 3). For this analytical method a $1 / \mathrm{C}^{\wedge} 2$ linear equation as the weighing factor was chosen to achieve a linear regression; the linear equation were as follows: IVA ( $\left.y=10^{7} x-96,473 ; R^{2}=0.999\right)$, M1 $\left(y=2 \times 10^{7} \mathrm{x}+2 \times 10^{6} ; \mathrm{R}^{2}=0.9961\right), \mathrm{M} 6\left(\mathrm{y}=5 \times 10^{6} \mathrm{x}+10^{6} ; \mathrm{R}^{2}=0.9898\right)$ and LUMA $\left(y=3 \times 10^{7} x+5 \times 10^{6} ; R^{2}=0.9954\right)$. The lower limit of detection (LOD) and quantification (LOQ) were established: for IVA LOD $2.50 \times 10^{-3} \mu \mathrm{g} / \mathrm{mL}$ and LOQ $7.57 \times 10^{-3} \mu \mathrm{g} / \mathrm{mL}$; for M1, LOD $4.57 \times 10^{-4} \mu \mathrm{g} / \mathrm{mL}$ and LOQ $1.38 \times 10^{-3} \mu \mathrm{g} / \mathrm{mL}$; for M6 LOD $5.86 \times 10^{-4} \mu \mathrm{g} / \mathrm{mL}$ and LOQ $1.78 \times 10^{-3} \mu \mathrm{g} / \mathrm{mL}$; for LUMA LOD was $6.08 \times 10^{-4} \mu \mathrm{g} / \mathrm{mL}$ and LOQ $1.84 \times 10^{-3}$ $\mu \mathrm{g} / \mathrm{mL}$ (Table 1). The combination of ACN/0.1\% formic acid provided optimal protein precipitation and excellent recovery of IVA, M1, M6 and LUMA from the plasma matrix. Recovery was $71.6 \%$ IVA, $84.2 \%$ M1, 82.2\% M6 and 101.2\% LUMA, respectively, indicating that IVA, M1, M6 and LUMA could be quantitatively recovered from plasma. The calibration range from 0.01 to $10 \mu \mathrm{g} / \mathrm{mL}$ is applicable for the use in clinical setting, in particular given that under ORKAMBI treatment very low concentrations of active IVA and M1 are observed in plasma (Figure 4 and Table 2), potentially due to the induction of CYP3A4 by LUMA which results in extensive IVA metabolism [16, 21].

On each analytical day ( 0 and 10 days) calibration curves were constructed using the relationship between the peak area ratios of IVA to IS and the calibration standard nominal concentrations of IVA, M1, M6 and LUMA. No tendency of degradation of the analytes was observed under the assay conditions, IVA, M1, M6 and LUMA concentrations remained stable following storage on the bench top for 10 days at $20^{\circ} \mathrm{C}$. Analytes stability in methanol and in human plasma were tested by placing aliquots of the working solution of analytes in methanol and preparations in human plasma into clear glass HPLC vials and storing them for 10 days at room temperature protected from light. Then samples were analysed against freshly prepared standards and the percent analyte remaining in the solution after the storage was calculated. For all analytes the determined values remained within $2 \%$ of the original values indicating a good stability (Table 1 ).

The clinical utility of the assay was demonstrated by its application to plasma and sputum samples from patients receiving KALYDECO or ORKAMBI oral therapy (Figure 4 and Table 2). The assay results revealed that the KALYDECO patient exhibited IVA plasma concentrations of $0.97 \mu \mathrm{g} / \mathrm{mL}$ at $2.5 \mathrm{~h}$ post the dose (IVA $150 \mathrm{mg} / \mathrm{q} 12 \mathrm{~h}$ ); M1 and M6 plasma concentrations were $0.50 \mu \mathrm{g} / \mathrm{mL}$ and $0.16 \mu \mathrm{g} / \mathrm{mL}$, respectively. Surprisingly, the patient receiving ORKAMBI (200 mg/q12 h LUMA-125 mg/q12 h IVA) displayed very low concentrations of IVA $(0.06 \mu \mathrm{g} / \mathrm{mL})$ and $\mathrm{M} 1(0.07 \mu \mathrm{g} / \mathrm{mL})$. The M6 concentration in the ORKAMBI patients was $0.15 \mu \mathrm{g} / \mathrm{mL}$, which is comparable to that of the KALYDECO patient [16]. However, relatively high concentrations of LUMA were detected $(4.42 \mu \mathrm{g} / \mathrm{mL})$. Moreover, the presence of appreciable amounts of IVA in the sputum of the KALYDECO patient, suggests the drug effectively distributes to the lungs. Notably, IVA was barely detectable in the sputum of the ORKAMBI patient.

Since the release of ORKAMBI, several red-flags have been raised that signal the clinical efficacy of this clever combination strategy maybe be limited due to antagonistic drug-drug 
interactions (ie a case of LUMA versus IVA) [13]. Such inhibitory interactions are especially concerning given that LUMA only partially restores (11-15\%) F508del-CFTR surface expression [13, 22, 23]. Various independent research groups have reported that IVA downregulates the restored F508del-CFTR whether it was modulated by LUMA or the investigational corrector VX-661 [14, 22, 23]. Matthes et al claimed that the inhibitory effects of IVA against the corrector function of LUMA only occurs at relatively high IVA concentrations $(5-10 \mu \mathrm{M}$, used in all of the reported cell culture studies) and that this inhibitory effect is unlikely to occur at the low (1.5-8.5 nM) free IVA concentrations achievable in vivo [14]. Lastly, in the FDA and EMA reports for ORKAMBI, Vertex indicates that LUMA may be an inducer of cytochrome P450 drug metabolizing enzymes and paradoxically IVA is a substrate of CYP3A4 [16, 21]. Moreover, the product information sheet for ORKAMBI strongly contraindicates co-administration of strong CYP3A4 inducers such as rifampin or St. John's Wort [18]. The latter would suggest another potential antagonistic drug-drug interaction between LUMA and IVA is at play where the former induces the metabolism of the latter. Notably, the very low concentrations of IVA we detected in the ORKAMBI patient would lend support to aforementioned CYP450 mediated issues. Overall, these factors may be compounding together to limit the clinical efficacy of ORKAMBI therapy.

\section{Conclusions}

Given the noted metabolism of IVA and possible antagonistic drug-drug interactions between IVA and LUMA, therapeutic drug monitoring in patient plasma is requisite to achieve optimizing dosage regimens for ORKAMBI therapy. The validated method described in this report allows the rapid quantification of IVA, M1, M6 and LUMA in plasma and sputum. The short run time is indispensable for the analysis of clinical samples. The deployment of this assay in the hospital setting will afford clinicians the rational to develop exposure-response relationships to maximize drug plasma levels and to achieve the best possible clinical efficacy. Also the assay may prove useful on the grounds of making the expensive KALYDECO and ORKAMBI therapy more cost effective, by potentially indicating the need for less frequent dosing. Albeit, the realization of all of these objectives will require PK/PD studies with a large patient cohort, for whom the present assay platform will be of considerable benefit.

\section{Acknowledgments}

J.L and T.V. are supported by the National Institute of Allergy and Infectious Diseases (NIAID) of the National Institutes of Health (R01 AI111965). The content is solely the responsibility of the authors and does not necessarily represent the official views of the National Institute of Allergy and Infectious Diseases or the National Institutes of Health. MC is an Australian NHMRC Principal Research Fellow. J. L. is an Australian National Health and Medical Research Council (NHMRC) Senior Research Fellow, and T. V. is an Australian NHMRC Industry Career Development Level 2 Research Fellow.

\section{References}

1. Bobadilla JL, Macek M Jr, Fine JP, Farrell PM. Cystic fibrosis: a worldwide analysis of CFTR mutations--correlation with incidence data and application to screening. Human mutation. 2002; 19:575-606. [PubMed: 12007216] 
2. Schneider EK, Huang JX, Carbone V, Baker M, Azad MA, Cooper MA, et al. Drug-drug plasma protein binding interactions of ivacaftor. Journal of molecular recognition: JMR. 2015; 28:339-48. [PubMed: 25707701]

3. Solomon, M. Cystic fibrosis-update on diagnosis and treatment from Cystic fibrosis etiology, diagnosisn and treatments. In: Leatte, PN., editor. Treatments for Cystic fibrosis. 2009.

4. Myers, LB. The role of adherence, importance and burden from Cystic fibrosis etiology, diagnosis and treatments. In: Leatte, PN., editor. Treatments for Cystic fibrosis: Nova Biomedical. 2009.

5. Tizzano EF, Buchwald M. CFTR expression and organ damage in cystic fibrosis. Annals of internal medicine. 1995; 123:305-8. [PubMed: 7541969]

6. O'Sullivan BP, Flume P. The clinical approach to lung disease in patients with cystic fibrosis. Seminars in respiratory and critical care medicine. 2009; 30:505-13. [PubMed: 19760538]

7. Ramsey BW, Davies J, McElvaney NG, Tullis E, Bell SC, Drevinek P, et al. A CFTR potentiator in patients with cystic fibrosis and the G551D mutation. The New England journal of medicine. 2011; 365:1663-72. [PubMed: 22047557]

8. Van Goor F, Hadida S, Grootenhuis PD, Burton B, Cao D, Neuberger T, et al. Rescue of CF airway epithelial cell function in vitro by a CFTR potentiator, VX-770. Proceedings of the National Academy of Sciences of the United States of America. 2009; 106:18825-30. [PubMed: 19846789]

9. Eckford PD, Li C, Ramjeesingh M, Bear CE. Cystic fibrosis transmembrane conductance regulator (CFTR) potentiator VX-770 (ivacaftor) opens the defective channel gate of mutant CFTR in a phosphorylation-dependent but ATP-independent manner. The Journal of biological chemistry. 2012; 287:36639-49. [PubMed: 22942289]

10. Jih KY, Hwang TC. Vx-770 potentiates CFTR function by promoting decoupling between the gating cycle and ATP hydrolysis cycle. Proceedings of the National Academy of Sciences of the United States of America. 2013; 110:4404-9. [PubMed: 23440202]

11. Wainwright CE, Elborn JS, Ramsey BW, Marigowda G, Huang X, Cipolli M, et al. LumacaftorIvacaftor in Patients with Cystic Fibrosis Homozygous for Phe508del CFTR. The New England journal of medicine. 2015; 373:220-31. [PubMed: 25981758]

12. Ferkol T, Quinton Paul. Precision Medicine: At What Price? American journal of respiratory and critical care medicine. 2015:192.

13. Cholon DM, Esther Charles R, Gentzsch Martina. Efficacy of lumacaftor-ivacaftor for the treatment of Cystic Fibrosis patients homozygous for the F508del-CFTR mutation. Expert Review of Precision Medicine and Drug Development. 2016

14. Matthes E, Goepp J, Carlile GW, Luo Y, Dejgaard K, Billet A, et al. Low free drug concentration prevents inhibition of F508del CFTR functional expression by the potentiator VX-770 (ivacaftor). British journal of pharmacology. 2016; 173:459-70. [PubMed: 26492939]

15. FDA. commitee FACBMV-FP-Ada. Sponsor Briefing Document: ORKAMBI (Lumacaftor/ Ivacaftor) for the Treatment of Cystic Fibrosis in Patients Age 12 Years and Older Who are Homozygous for the F508del Mutation in the CFTR Gene. VERTEX Pharmaceuticals Incorporated; 2015. p. 98

16. Assessment report ORKAMBI (ivacaftor/lumacaftor) European medicines agency EMEA/H/C/ 003954/0000. European medicines agency EMEA/H/C/003954/00002015.

17. Yildiz A, Guleryuz S, Ankerst DP, Ongur D, Renshaw PF. Protein kinase C inhibition in the treatment of mania: a double-blind, placebo-controlled trial of tamoxifen. Arch Gen Psychiatry. 2008; 65:255-63. [PubMed: 18316672]

18. VERTEX. Vertex prescribing infomation. 2015. Highlights of prescribing information ORKAMBI tablets for oral use.

19. FDA U. Guidance for Industry: Bioanalytical Method Validation. 2001.

20. Su Q, Li J, Ji X, Li J, Zhou T, Lu W, et al. An LC-MS/MS method for the quantitation of cabozantinib in rat plasma: application to a pharmacokinetic study. Journal of chromatography B, Analytical technologies in the biomedical and life sciences. 2015; 985:119-23. [PubMed: 25678398]

21. Wainwright CE. Ivacaftor for patients with cystic fibrosis. Expert review of respiratory medicine. 2014; 8:533-8. [PubMed: 25148205] 
22. Cholon DM, Quinney NL, Fulcher ML, Esther CR Jr, Das J, Dokholyan NV, et al. Potentiator ivacaftor abrogates pharmacological correction of DeltaF508 CFTR in cystic fibrosis. Science translational medicine. 2014; 6:246ra96.

23. Veit G, Avramescu RG, Perdomo D, Phuan PW, Bagdany M, Apaja PM, et al. Some gating potentiators, including VX-770, diminish DeltaF508-CFTR functional expression. Science translational medicine. 2014; 6:246ra97. 


\section{Highlights}

- We are the first to develop and validate a sensitive, rapid and novel HPLC and LC-MS methods for the rapid detection and quantification of ivacaftor and its major metabolites hydroxymethyl-ivacaftor M1 (active) and ivacaftor carboxylate M6 (inactive), and lumacaftor for the first time in the plasma and sputum of CF patients.

- $\quad$ Our findings shed light upon the context of the potential antagonistic drugdrug interactions between lumacaftor and ivacaftor.

- This novel and reliable method offers a simple and sensitive approach for therapeutic drug monitoring of KALYDECO and ORKAMBI in plasma and sputum.

- The introduction of the novel assay into the clinical setting will facilitate pharmacokinetics/pharmacodynamic analysis and assist clinicians to develop exposure-response relationships for these breakthrough CF drugs. 


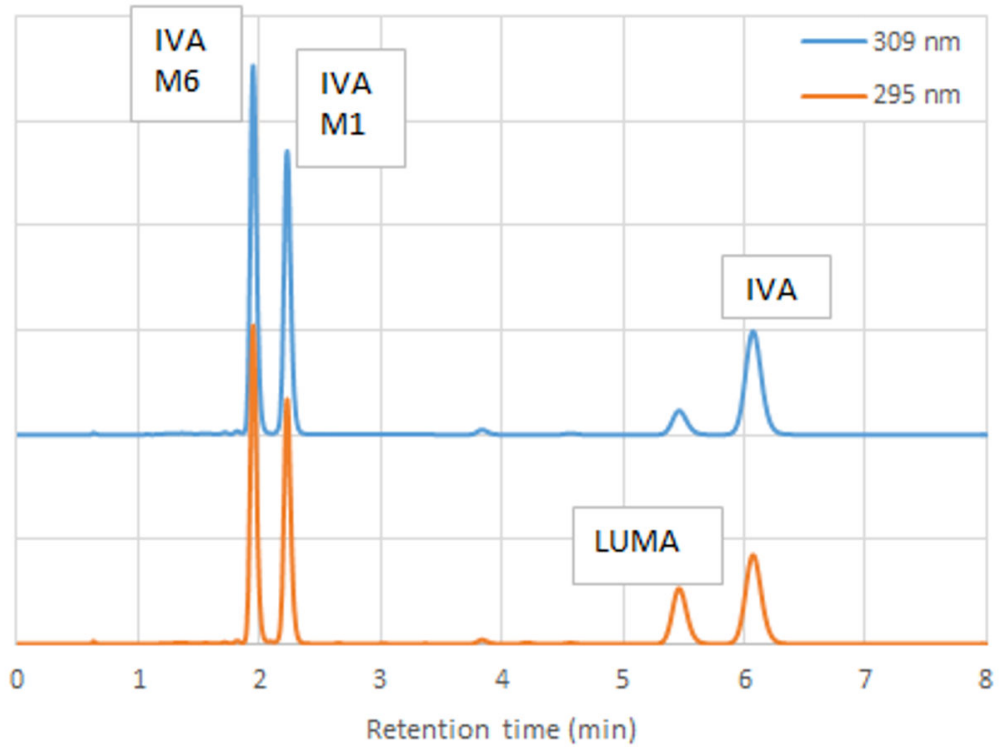

Figure 1.

Representative UV chromatograms of each analyte (M6, M1 and IVA and LUMA) at 10 $\mu \mathrm{g} / \mathrm{mL}$. Quantification of IVA (also M1 and M6) and LUMA was performed at $309 \mathrm{~nm}$ and $295 \mathrm{~nm}$, respectively. 


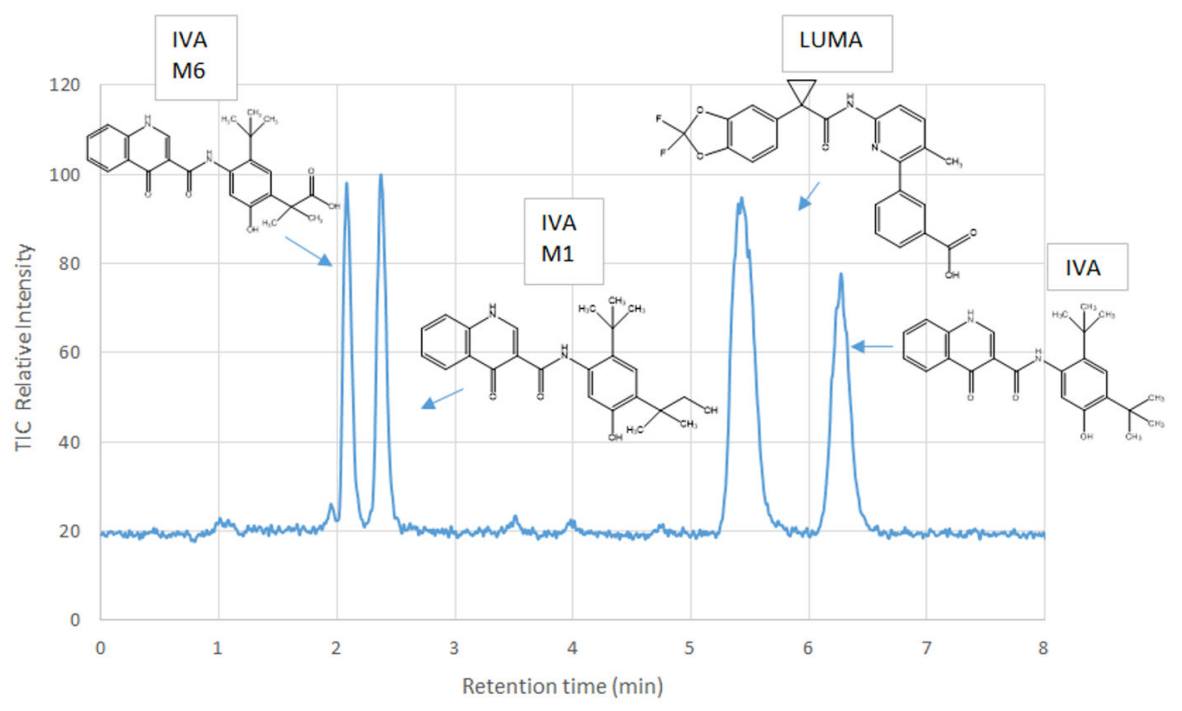

Figure 2.

A representative LC-MS Chromatogram of human plasma spiked with $10 \mu \mathrm{g} / \mathrm{mL}$ of M6, M1, LUMA and IVA. The ion transitions of $m / z 392.49 \rightarrow 393, m / z \quad 408.49 \rightarrow 409, m / z$ $422.47 \rightarrow 423$ and $\mathrm{m} / z 452.40 \rightarrow 453$ were used for MS/MS monitoring of IVA, M1, M6 and LUMA, respectively. 

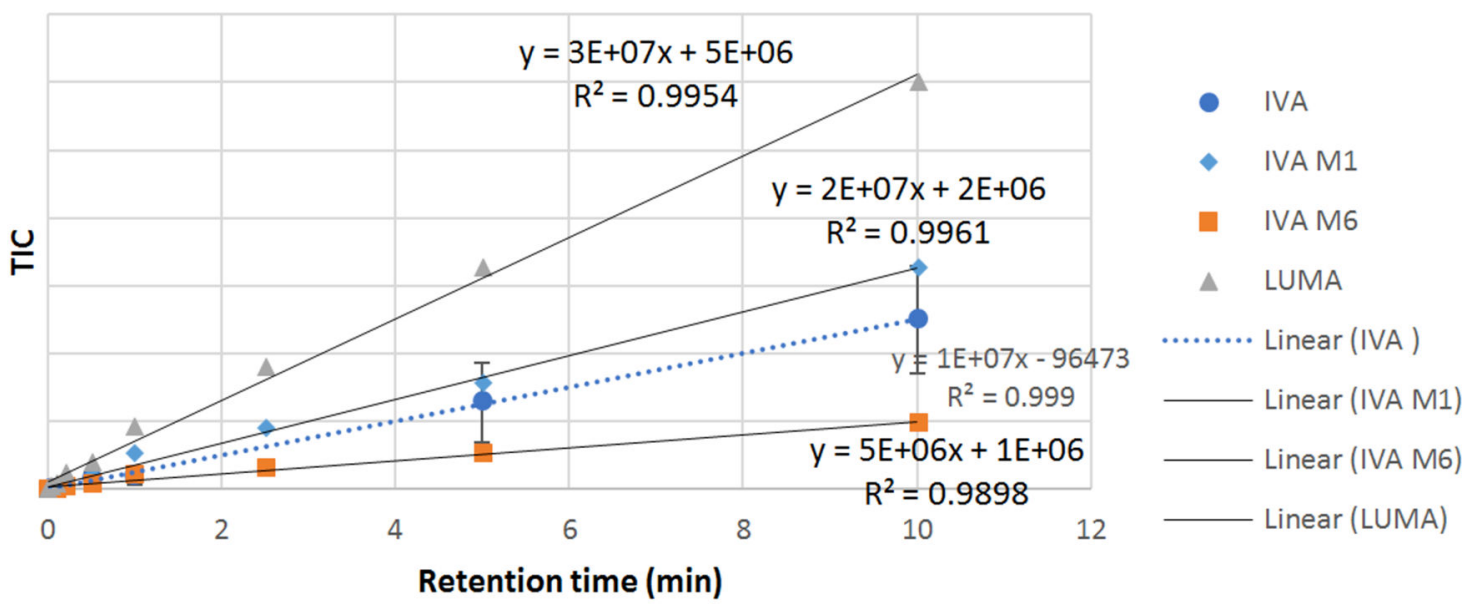

Figure 3.

Calibration plot of all four analytes (IVA, M1, M6 and LUMA) using $1 / \mathrm{C}^{\wedge} 2$ for weighing factor to achieve a linear regression. The linear equations were as follows: IVA $\left(y=10^{7} \mathrm{x}\right.$ -96,473; $\left.\mathrm{R}^{2}=0.999\right), \mathrm{M} 1\left(\mathrm{y}=2 \times 10^{7} \mathrm{x}+2 \times 10^{6} ; \mathrm{R}^{2}=0.9961\right), \mathrm{M} 6\left(\mathrm{y}=5 \times 10^{6} \mathrm{x}+10^{6} ; \mathrm{R}^{2}=0.9898\right)$ and LUMA $\left(\mathrm{y}=3 \times 10^{7} \mathrm{x}+5 \times 10^{6} ; \mathrm{R}^{2}=0.9954\right)$. 


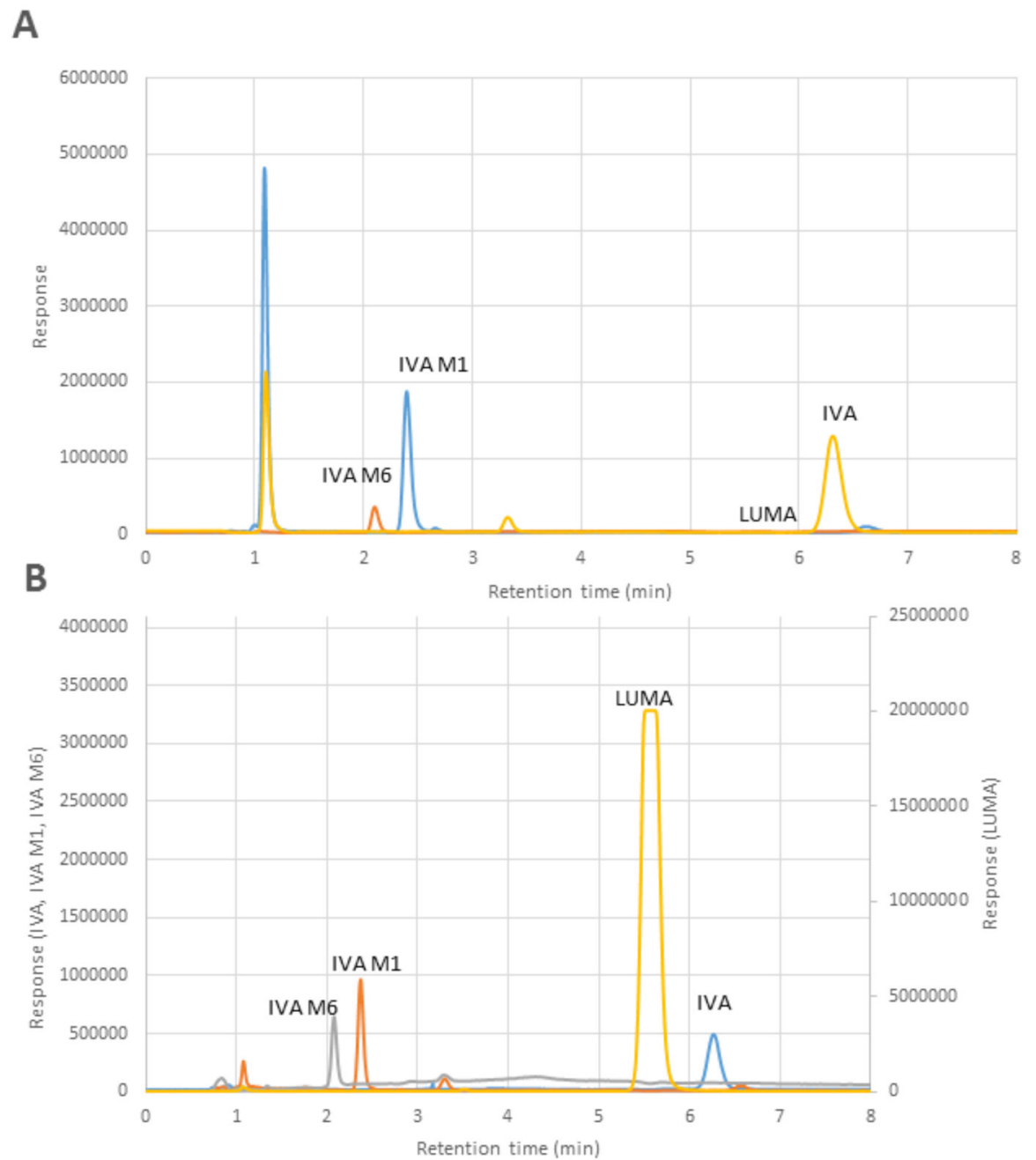

Figure 4.

LC-MS chromatogram of the plasma patient receiving (A) KALYDECO or (B) ORKAMBI. The LC retention times were as follows IVA $6.2 \mathrm{~min}$, M1 $2.4 \mathrm{~min}, \mathrm{M} 62.1 \mathrm{~min}$ and LUMA $5.4 \mathrm{~min}$. 


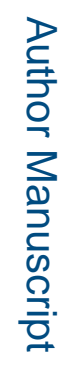

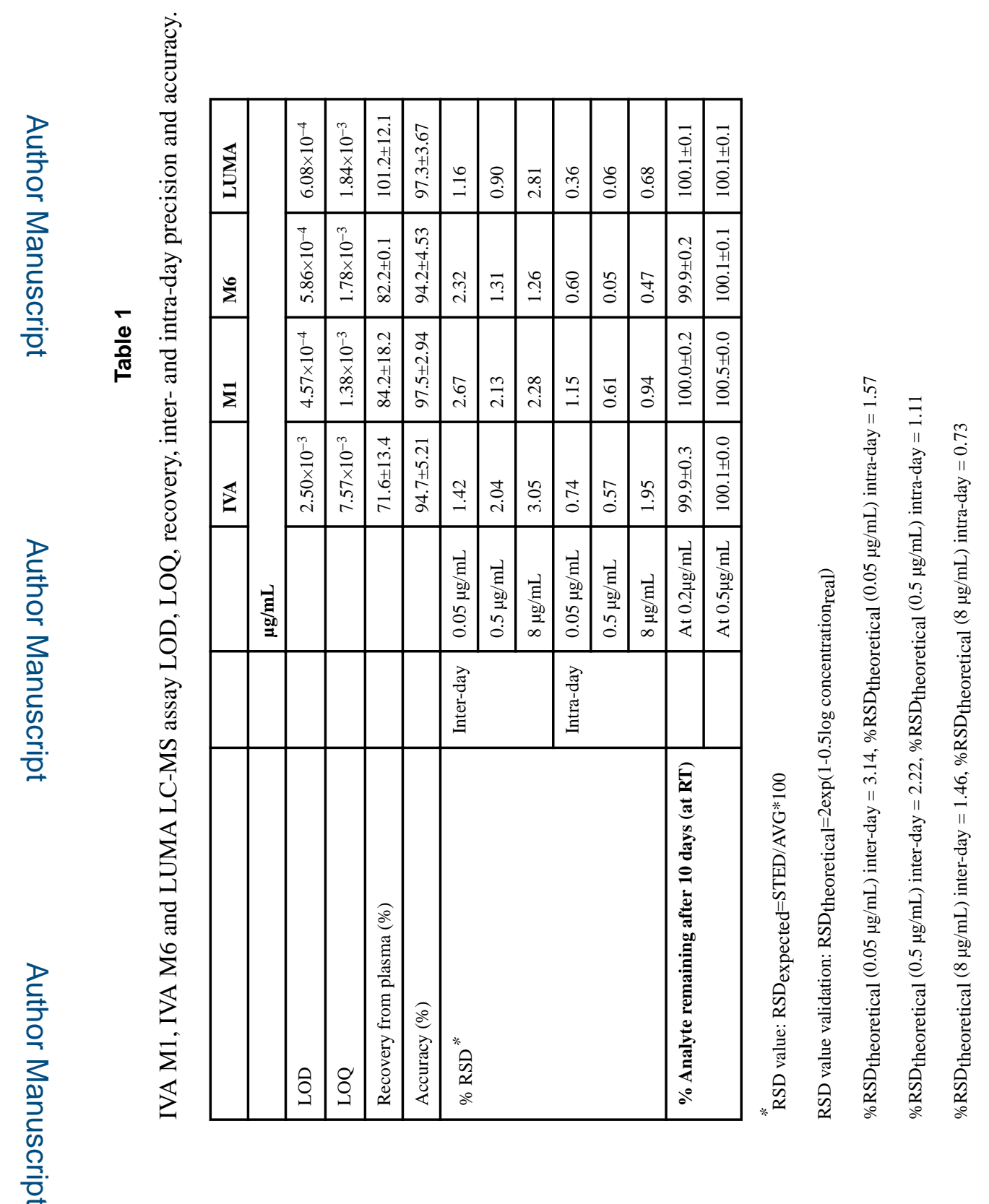

J Chromatogr B Analyt Technol Biomed Life Sci. Author manuscript; available in PMC 2017 December 01. 


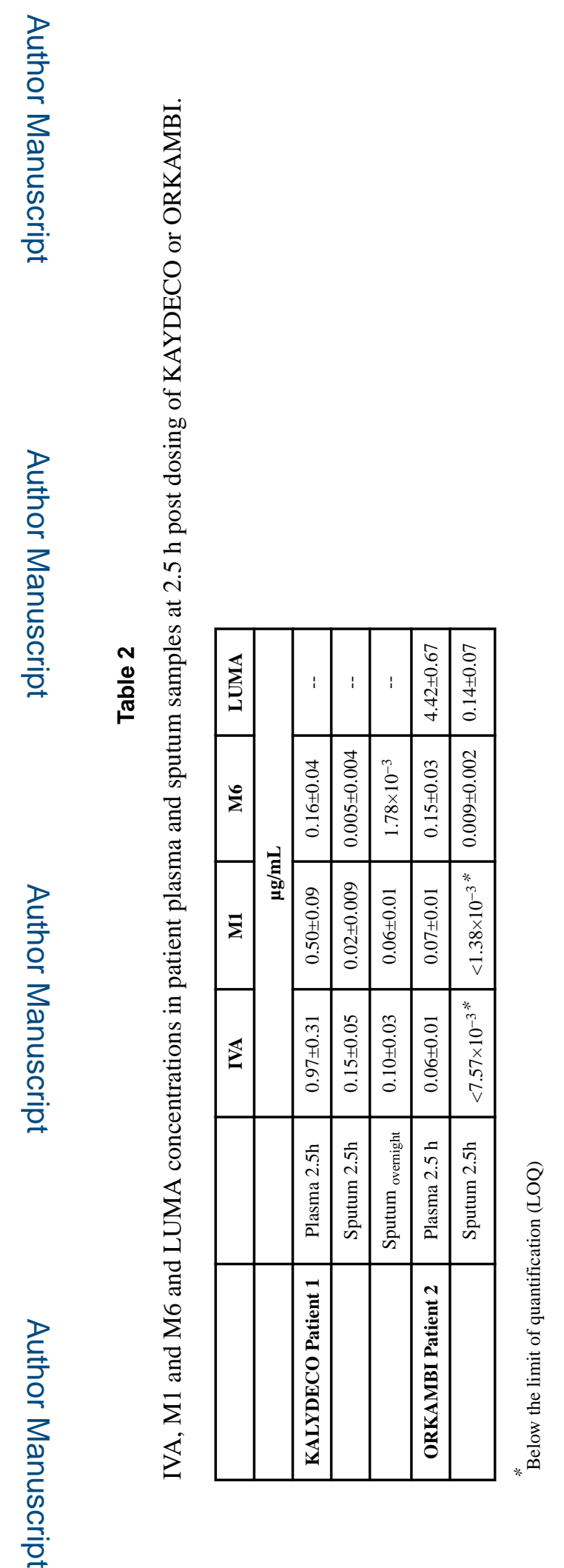

